\title{
Staphylinidae e Silphidae (Coleoptera) como Potenciais Famílias Bioindicadoras de Qualidade Ambiental
}

\author{
Fabiana dos Santos Fernandes \\ Universidade Severino Sombra, CECETEN, Discente de Graduação em \\ Ciências Biológicas, Laboratório de Biomonitoramento Ambiental \\ fabianas.fernandes@hotmail.com \\ Sueyla da Silva Alves \\ Universidade Severino Sombra, CECETEN, Discente de Graduação em \\ Ciências Biológicas, Laboratório de Biomonitoramento Ambiental \\ sueyla_alves@hotmail.com \\ Hélio Freitas Santos \\ Universidade Severino Sombra, Discente de Mestrado em Ciências \\ Ambientais, Laboratório de Biomonitoramento Ambiental \\ prof.helioizma@gmail.com / admin@izma.org.br \\ William Costa Rodrigues \\ Universidade Severino Sombra, Mestrado em Ciências Ambientais, \\ Laboratório de Biomonitoramento Ambiental \\ wcrodriguesdebras.bio.br
}

Resumo: O presente estudo teve como objetivo levantar a biodiversidade de coleópteros do IZMA (Eng. Paulo de Frontin - RJ), compreender suas relações com as composições florísticas e buscar identificar potenciais bioindicadores. Foram realizadas quatro coletas, em oito pontos distintos, de abril de 2008 a março de 2009, com utilização de armadilhas pitfall. Os insetos foram triados, identificados, e armazenados no Laboratório de Monitoramento Ambiental - USS; os dados foram analisados por meio da Diversidade e Dominância de Simpson e Similaridade de Cluster. Capturaram-se 1990 coleópteros (15 familias), com maior ocorrência de Staphylinidae no interior da mata. Os pontos V e VI foram os mais similares e Silphidae, em áreas intermediárias; os pontos VII e VIII, mais similares. A $1^{a}$ coleta apresentou maior dominância; a $4^{a}$ coleta, maior diversidade. O ponto IV obteve maior abundância; o V, maior dominância; os pontos I e III, maior diversidade; os pontos III e VII, maior similaridade. Os pontos III, IV, VI, e VII mostraram-se os mais conservados, Staphylinidae e Silphidae foram apontados como potenciais bioindicadores, por apresentar sensibilidade às modificações do ambiente.

Palavras-chave: Bioindicadores. Fauna Edáfica. Serrapilheira. Pitfall. 


\title{
Staphylinidae and Silphidae (Beetles) as Potencial Biomakers Families Of Environmental Quality
}

\begin{abstract}
This study aimed to display the biodiversity of beetles at IZMA (Eng. Paulo de Frontin, RJ), to understand their relations with floristic composition, seeking to identify potencial biomakers. Four collections were held in eight different points, from April 2008 to march 2009, using pitfall traps. The insects were sorted, labeled and stored in the Environmental Monitoring Laboratory and the data analyzed by Simpson's Dominance and Diversity, and Similarity Cluster. Were captured 1990 beetles (15 families), with higher occurrence of: Staphylinidae in the forest interior, points $V$ and VI were the most similar ones; and Silphidae in intermediate areas, being the points VII and VIII more similar to each other. The first sample showed a higher dominance, the fourth collection, greater diversity. Point IV was the most abundant; point V, the highest dominance; points I and III, the highest similarity. Points III, IV, VI and VII proved to be the most highly conserved, pointing Staphylinidae and Silphidae as potential biomarkers, for sensitivity to changes in the present environment.
\end{abstract}

Keywords: Biomakers. Edafic fauna. Litter. Pitfall.

\section{Introdução}

O solo está entre os mais complexos sistemas biológicos do globo terrestre. Além de garantir lugar para a vida de muitos organismos, possui uma estreita relação com as cadeias alimentares das quais depende a maioria, senão todos os organismos terrestres, pois é o substrato de sustentação dos vegetais (Stork e Eggleton, 1992).

A composição da fauna do solo reflete o funcionamento do ecossistema, devido à sua íntima associação aos processos do sistema serrapilheira-solo e sua grande sensibilidade às modificações ambientais (Correia e Pinheiro, 1999). Como a fauna do solo e da serrapilheira apresentam alta diversidade e rápida capacidade de reprodução, são excelentes bioindicadores, e suas propriedades ou funções indicam e determinam a qualidade ou o nível de degradação do solo (Knoepp et al., 2000).

A preocupação com questões ambientais aumentou a demanda por bioindicadores capazes de refletir seu ambiente. Entre esses organismos, os insetos podem contribuir para uma avaliação prática do grau de sustentabilidade (Lopes, 2008). Segundo Tylianakis et al. (2004), insetos indicadores tornam-se particularmente úteis, porque representam mais da metade de todas as espécies e sua diversidade permite avaliar a diferença entre habitat em escala requintado aceitáveis.

Muitos insetos podem ser usados como bioindicadores da poluição ambiental. As formigas têm sido usadas para medir as concentrações de poluentes nas florestas boreais e Austrália e são atualmente utilizados para monitorar os ecossistemas perturbados. As abelhas são consideradas um dos bioindicadores mais eficientes. São usadas para monitorar traços de 
metais em ambientes urbanos e a radioatividade após o desastre de Chernobyl, efeitos de pesticidas e herbicidas, resíduos industriais e poluentes (Urbini et al., 2006).

Além da facilidade de amostragem, os bioindicadores, devem ter uma resposta já conhecida à alteração ambiental, bem como responder de maneira clara ao distúrbio, e informar sobre a estrutura, o funcionamento, e a composição do sistema ecológico, devendo ser monitorados em distúrbios ambientais a curto e longo prazo (Dale e Beyeler, 2001). Os mais importantes indicadores são os insetos, por ser o grupo mais diverso em número de espécies, e pela facilidade de amostragem; a diversidade de insetos edáficos pode revelar o nível de qualidade ambiental, a partir do qual podem ser determinadas intervenções a fim de manter, recuperar ou restaurar a sanidade ambiental, atingindo a sustentabilidade ecológica dos ecossistemas (Wink, 2001).

Invertebrados são mais severamente afetados e rapidamente do que outros taxa por mudanças na paisagem. Os insetos são responsáveis por muitos processos no ecossistema e sua perda pode ter efeitos negativos sobre comunidades inteiras. Assim, uma forte compreensão das respostas inseto à atividade humana é necessária tanto para apoiar decisões de política para a conservação e para avaliar as consequências funcionais da perturbação humana sobre os ecossistemas (Nicholsa et al., 2007).

A ordem Coleoptera constitui-se no maior agrupamento de animais que se conhece, pois perfaz cerca de $23 \%$ de todos os animais conhecidos e $35 \%$ do total de insetos (Buzzi e Miyazaki, 1999). Algumas famílias de Coleoptera possuem atributos desejáveis para serem incluídas como bioindicadores, pois são grupos que possuem boa parte das espécies com alta fidelidade ecológica, são altamente diversificados taxonomicamente e ecologicamente, facilmente coletáveis em grandes quantidades, e funcionalmente importantes nos ecossistemas (Brown, 1991).

Os estudos da fauna de Coleoptera envolvendo apenas as famílias ou espécies mais abundantes são uma forma de minimizar as dificuldades de identificação dos táxons mais raros, e acelerar as análises; é importante que se procure levantar o maior número de informações sobre as diferentes relações entre essa fauna e o ambiente, neste nível de organização taxonômica, para consolidar o grupo como possível indicador de condições ambientais em áreas florestadas (Marinoni e Ganho, 2003).

O presente estudo tem como objetivo levantar a biodiversidade de coleópteros de um fragmento de Mata Atlântica, localizado no IZMA, Eng. Paulo de Frontin - RJ; procurando evidenciar as relações entre as famílias mais abundantes e as diferentes composições florísticas, buscando identificar grupos de potenciais bioindicadores, além de contribuir para o conhecimento da fauna local.

\section{Material e Métodos}

O estudo foi realizado no Instituto Zoobotânico de Morro Azul (IZMA), localizado no município de Eng. ${ }^{\circ}$ Paulo de Frontin - RJ, geograficamente situado a $22^{\circ} 29^{\prime}$ S e $43^{\circ} 34^{\prime}$ W, com altitude variando entre 671 a 825 metros, em um fragmento de Floresta Atlântica, com uma área de aproximadamente 19 ha, onde existe uma trilha ecológica - Trilha dos Quatis - com extensão de 2200 metros (Figura 1). 
Fabiana dos Santos Fernandes - Sueyla da Silva Alves - Hélio Freitas Santos - William Costa Rodrigues

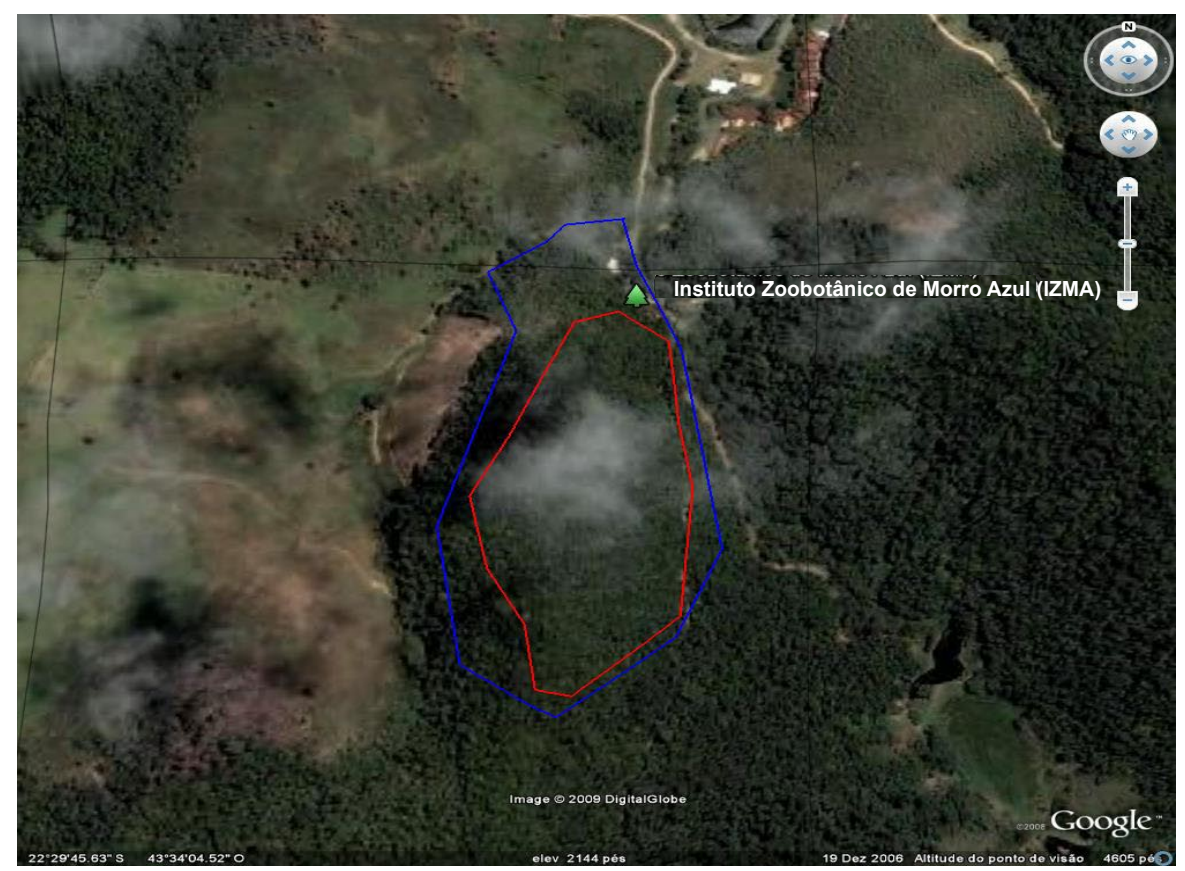

Figura 1. Área do Instituto Zoobotânico de Morro Azul. O Polígono vermelho delimita a área de estudo dentro do instituto, o polígono azul delimita a área total.

Foram realizadas quatro coletas, trimestralmente, de abril de 2008 a março de 2009, abrangendo as quatro estações num período de um ano.

Foram distribuídos oito pontos (I a VIII) de coleta, com área de $30 \mathrm{~m}^{2}$ cada, ao longo da Trilha dos Quatis, dispostos em seis altitudes diferentes, considerando a composição florística, o nível da serrapilheira (NS1: 0 a $5 \mathrm{~cm}, \mathrm{NS} 2: 6$ a $15 \mathrm{~cm}, \mathrm{NS} 3:>15 \mathrm{~cm}$ ) e a luminosidade:

I - $\quad$ Borda da mata, com vegetação intermediária, NS1;

II - Área de transição, com vegetação rasteira (aspecto descampado), NS1;

III - Área intermediária, com vegetação rasteira (presença de arbustos e árvores), NS2;

IV - Interior da mata, início da vegetação densa, NS3;

V - Interior da mata, com vegetação rasteira, (presença de gramíneas e árvores espaçadas, NS1;

VI - Interior da mata, com vegetação densa, NS3;

VII - Área intermediária, com vegetação rasteira (presença de árvores grandes), NS2;

VIII - Área de transição, próxima à borda da mata (presença de árvores e arbustos), NS2.

Para a captura dos insetos foram utilizadas seis armadilhas de solo do tipo pitfall para cada ponto, estando distantes entre si $10 \mathrm{~m}$, e $5 \mathrm{~m}$ da trilha, aproximadamente, permanecendo 48 $\mathrm{h}$ no ambiente. A armadilha pitfall consiste em um pote plástico com altura de $15 \mathrm{~cm}, 12$ 
cm de diâmetro, $500 \mathrm{~mL}$ de volume; contendo $150 \mathrm{~mL}$ de fixador (água 90\%, detergente neutro 10\% e formol 1\%) ( Figura 2A). Para evitar as inundações das armadilhas, devido à precipitação pluviométrica, foi instalada uma cobertura de arame e plástico (Figura 2B).

Os espécimes foram levados ao Laboratório de Monitoramento Ambiental - USS, onde foram triados, sendo armazenados em tubos de Eppendorf contendo álcool a $70 \%$. Posteriormente, os insetos foram identificados em nível de ordem, e os coleópteros, em nível de família; utilizando chave dicotômica, e auxílio de Lupa, com aumento de $40 \mathrm{x}$.

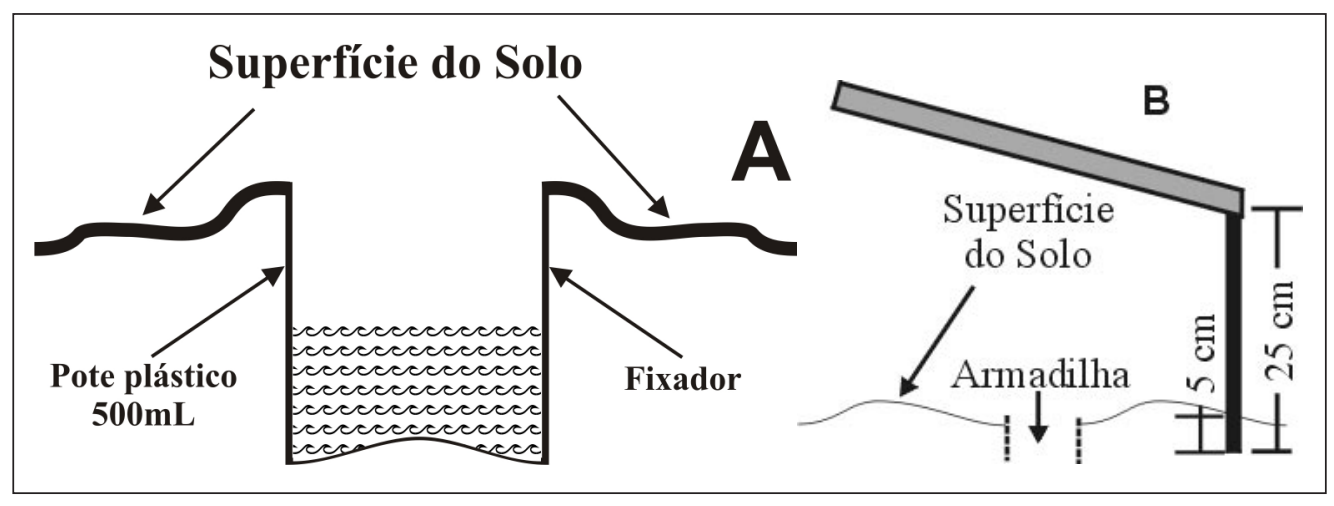

Figura 2. Armadilha de solo (pitfall), adaptada de Majer (1978) e Castro et al (1989). Armadilha de solo com fixador; B - Cobertura contra chuva fixada acima da armadilha.

A Diversidade e a Dominância das famílias foi estimada mediante índices de Simpson (Brower et al, 1997), utilizando o software DivEs - Diversidade de Espécies (Rodrigues, 2005). A Similaridade foi avaliada por análise de Cluster, com distância euclidiana e com ligação completa (Complete Linkage), tomando os pontos e suas repetições como tratamentos independentes.

\section{Resultados e Discussão}

Foram capturados 1990 coleópteros $(21,57 \%)$ no total das quatro coletas, distribuídos em 15 famílias, sendo Staphylinidae, e Silphidae as mais abundantes $(95,53 \%)$, enquanto as demais famílias (Scarabeidae, Limexylidae, Curculionidae, Scolytidae, Carabidae, Gyrinidae, Chrisomelidae, Bruchidae, Coccinelidae, Passalidae, Lucanidae, Elateridae e Bostrichidae), representaram apenas $4,47 \%$ do total de coleópteros coletados. Segundo Dummel et al (2011) do total de famílias de Coleoptera capturadas, 12 foram classificadas como comuns e as demais 14 como raras, dentre as famílias Staphylinidae foi considerada como comum a uma das duas área estudadas. Quando considerada a exclusividade nas áreas estudadas os autores afirmam que Silphidae é exclusiva de uma área e Staphylinidae de outra.

A $1^{\text {a }}$ coleta foi a mais abundante $(36,53 \%)$, realizada no mês central do outono, período em que há um aumento da serrapilheira; enquanto a $2^{\mathrm{a}}$ coleta foi a menos abundante (7,34\%), realizada no período do inverno. Essa variação indica que as coletas podem ter sofrido interferência a sazonalidade, estando vinculado a condições abióticas do próprio 
ambiente na época e estação climática.

A maioria das famílias consideradas comuns está entre os coleópteros que apresentam o maior número de espécies, como Carabidae, Cerambycidae, Chrysomelidae, Curculionidae, Elateridae, Scarabaeidae e Staphylinidae (Ianuzzi et al., 2003). As demais famílias comuns podem estar correspondendo às adaptações que possuem para os ambientes estudados (Dummel et al., 2011).

O ponto IV foi o que apresentou maior abundância, com 19,25\% do total das quatro coletas; seguida dos pontos III e VII, com $15,88 \%$ e 13,82\% de abundância, respectivamente. O ponto II foi o que apresentou menor abundância, com um total de 4,92\% (Tabela 1), provavelmente por ser uma área impactada, apresentando aspecto descampado.

A ocorrência das famílias Staphylinidae (56,28\%) e Silphidae (39,52\%) em todos os pontos foi superior em relação a todas as outras famílias de coleópteros capturados (Figura 3). Segundo Freitas et al (2005), aproximadamente metade das famílias de Staphylinidae é composta por habitantes da serrapilheira, formando um dos mais importantes componentes da fauna de solo, sendo um dos grupos mais bem representados em levantamentos deste ambiente.

Tabela 1. Frequência das famílias de Coleoptera, distribuído entre os pontos, coletados no período de abril de 2008 a março de 2009, no IZMA, Eng. Paulo de Frontin, RJ.

\begin{tabular}{|c|c|c|c|c|c|c|c|c|c|c|}
\hline Família & $\begin{array}{c}\text { Ponto } \\
\text { I }\end{array}$ & $\begin{array}{c}\text { Ponto } \\
\text { II }\end{array}$ & $\begin{array}{c}\text { Ponto } \\
\text { III }\end{array}$ & $\begin{array}{l}\text { Ponto } \\
\text { IV }\end{array}$ & $\begin{array}{c}\text { Ponto } \\
\text { V }\end{array}$ & $\begin{array}{c}\text { Ponto } \\
\text { VI }\end{array}$ & $\begin{array}{c}\text { Ponto } \\
\text { VII }\end{array}$ & $\begin{array}{c}\text { Ponto } \\
\text { VIII }\end{array}$ & Total & $\%$ \\
\hline Staphylinidae & 111 & 70 & 178 & 271 & 157 & 155 & 116 & 62 & 1120 & 56,28 \\
\hline Silphidae & 84 & 22 & 120 & 98 & 51 & 99 & 151 & 156 & 781 & 39,25 \\
\hline Outros & 10 & 6 & 18 & 14 & 10 & 11 & 8 & 12 & 89 & 4,47 \\
\hline Total & 205 & 98 & 316 & 383 & 218 & 265 & 275 & 230 & 1990 & 100 \\
\hline$\% \%$ & 10,30 & 4,92 & 15,88 & 19,25 & 10,95 & 13,32 & 13,82 & 11,56 & 100 & \\
\hline
\end{tabular}

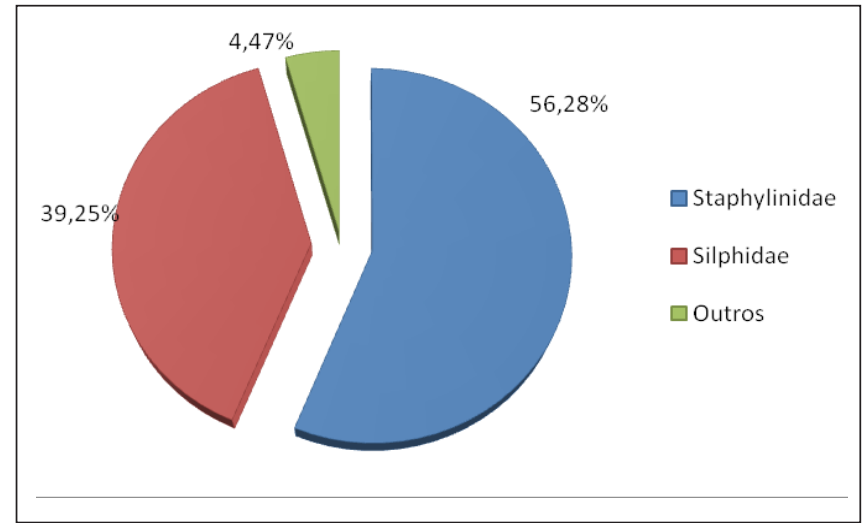

Figura 3. Percentual das famílias mais abundantes de Coleoptera, capturados no período de abril de 2008 a março de 2009, no IZMA, Eng. Paulo de Frontin - RJ. 
As áreas de interior (pontos IV, V, e VI) apresentaram maior ocorrência da família Staphylinidae, enquanto as áreas intermediárias (pontos III, VII, e VIII) apresentaram maior ocorrência da família Silphidae. Este resultado sugere que a primeira família tem maior afinidade com solos mais sombreados, contendo maior quantidade de serrapilheira (NS3), enquanto a segunda, parece preferir solos com maior luminosidade, e quantidade média de serrapilheira (NS2).

$\mathrm{O}$ ponto $\mathrm{V}$ difere das outras áreas de interior por apresentar uma vegetação de sub-bosque dominante, com presença, principalmente, da espécie Hypolytrum sp., vegetação arbórea espaçada, e pouca serrapilheira (NS1). O fato de essa área - está entre duas outras mais preservadas, com maior abundância de Staphylinidae, com certeza, influenciou o resultado; pois nas áreas mais próximas à borda, também apresentando pouca serrapilheira, tendo aspecto descampado (pontos I, II, VII, e VIII) houve declínio dessa população, chegando a menos da metade de sua abundância. Em Marinoni e Ganho (2003) foi constatada maior abundância de Staphylinidae em área com menor sombreamento, dossel mais aberto, presença de gramíneas e plantas arbustivas, mostrando certa tendência da família em áreas com essas características. No entanto, essa preferência pode ser resultante de uma combinação de fatores favoráveis a manutenção e sobrevivência destes insetos nesse ambiente, por exemplo, a composição da serrapilheira de áreas próximas à borda da mata, difere da composição da serrapilheira de áreas de interior, seja por características orgânicas, seja pela umidade. A composição do solo também é um fator de influência à abundância de coleópteros. Dunxião et al., (1999) relataram que insetos das famílias Staphylinidae e Scarabeidae estão relacionados a solos contendo concentrações de potássio e fósforo, além de serem frequentes em solos contendo material orgânico.

No que refere à família Silphidae, percebe-se haver preferência por uma vegetação intermediária, com presença de árvores e arbustos, além de temperaturas mais altas, fato também comprovado por ter havido maior ocorrência nas coletas da primavera, e início do outono/final do verão ( $3^{\mathrm{a}}$ e $4^{\mathrm{a}}$ respectivamente). Em levantamentos feitos no Paraná, em fragmentos de área secundária em diferentes estágios de sucessão, utilizando armadilha pitfall com diferentes tipos de iscas, a abundância de Silphidae não foi significativa $(<1 \%)$ (Petroni, 2008), o oposto encontrado no presente estudo, que obteve 39,52\% de ocorrência dessa família. Em um levantamento em Goiás, houve representatividade da família em área de mata semicaducifólia, não aparecendo em área de pastagem (Santos e Silva, 2004).

Segundo Costa Lima (1952), a família Staphylinidae possui hábito saprófago, mas algumas espécies são fungívoras e outras fitófagas, sendo habitualmente predadores; quanto a Silphidae, a maioria das espécies é associada a hábitos necrófagos, sendo que há espécies saprófagas e fungívoras. Os besouros da família Scarabaeidae são detritívoros, promovendo a remoção e reingresso da matéria orgânica no ciclo de nutrientes (Milhomen et al, 2003). Odum (1983) classifica como detritívoros todos os invertebrados do solo. Marinoni e Dutra (1997) constataram que os coleópteros herbívoros predominam em áreas degradadas, em início de regeneração, enquanto os grupos detritívoros e fungívoros predominam em áreas conservadas.

Considerando que a maioria das famílias de Staphylinidae e Silphidae encontradas no 
Staphylinidae e Silphidae (Coleoptera) como Potenciais Famílias Bioindicadoras de Qualidade Ambiental

Fabiana dos Santos Fernandes - Sueyla da Silva Alves - Hélio Freitas Santos - William Costa Rodrigues

fragmento, é de hábito detritívoro, pois foram coletados utilizando armadilha de solo, além de Scarabaeidae ( $3^{\mathrm{a}}$ família mais abundante), fazendo-se necessário ponderar que não se pode generalizar, pois em uma família podem existir vários grupos tróficos; os pontos III, IV, VI e VII, seriam os mais conservados, já que neles houve predominância dessas famílias, estando de acordo com a caracterização inicial das áreas (Figura 4).

Quando avaliada a Similaridade mediante análise de Cluster, entre os pontos; relacionada à família Silphidae, verificou-se que os pontos VII e VIII são os mais similares (Figura 5), mostrando uma fidelidade da família a esses pontos, nos quais apresentaram maior abundância. Enquanto, quando se trata da família Staphylinidae, os pontos V e VI apresentaram-se com maior Similaridade (Figura 6), já que o ponto IV foi muito mais abundante que os outros, ficando distante destes. Em relação às demais famílias, os pontos II e VII foram mais similares (Figura 7).

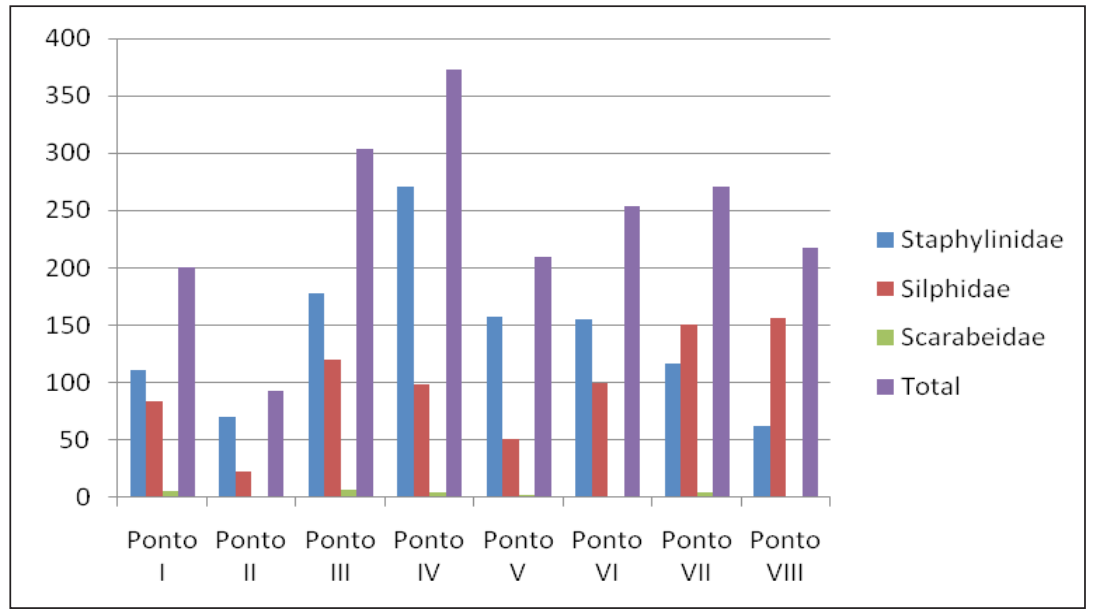

Figura 4. Distribuição entre os pontos de coleta das famílias de coleópteros mais abundantes, consideradas "detritívoras", capturadas no período de abril de 2008 a março de 2009, no IZMA, Eng. Paulo de Frontin - RJ.

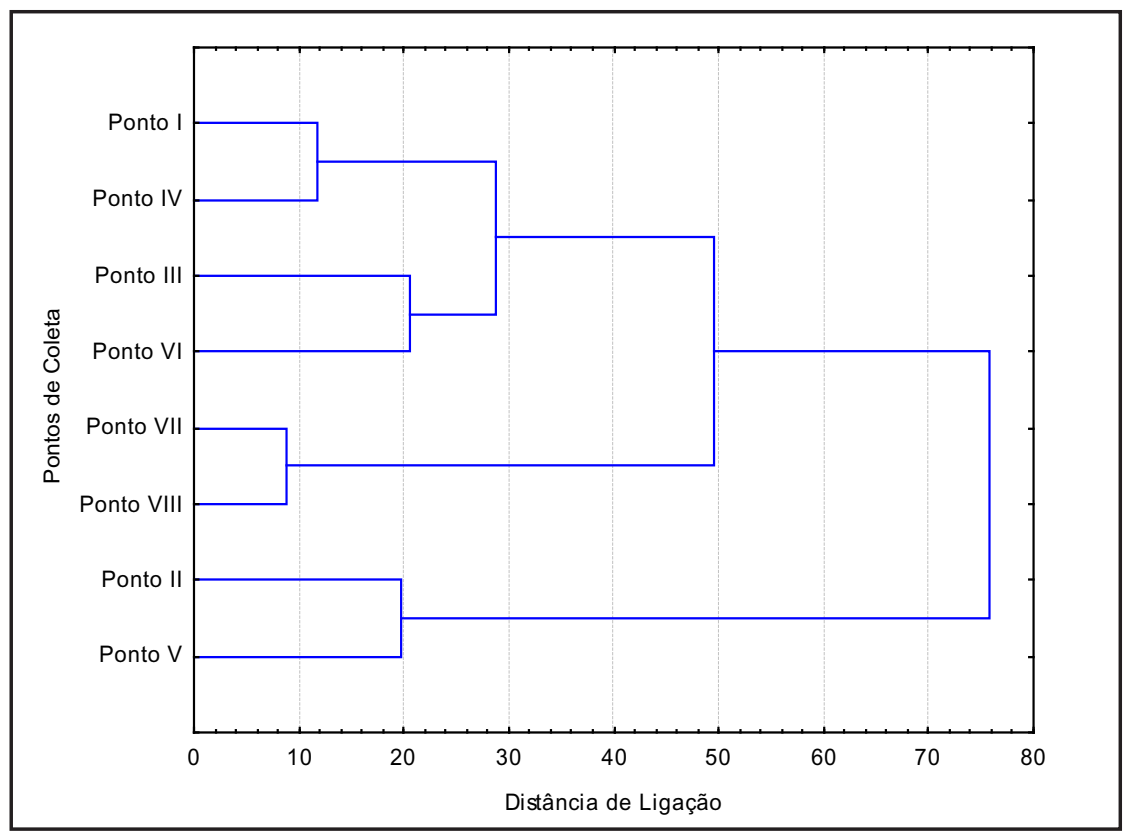

Figura 5. Diagrama de árvore da família de Silphidae entre os oito pontos de coleta, baseado na análise Cluster (Complete Linkage), no período de abril de 2008 e março de 2009, no IZMA, Eng. Paulo de Frontin - RJ. 
Staphylinidae e Silphidae (Coleoptera) como Potenciais Famílias Bioindicadoras de Qualidade Ambiental

Fabiana dos Santos Fernandes - Sueyla da Silva Alves - Hélio Freitas Santos - William Costa Rodrigues

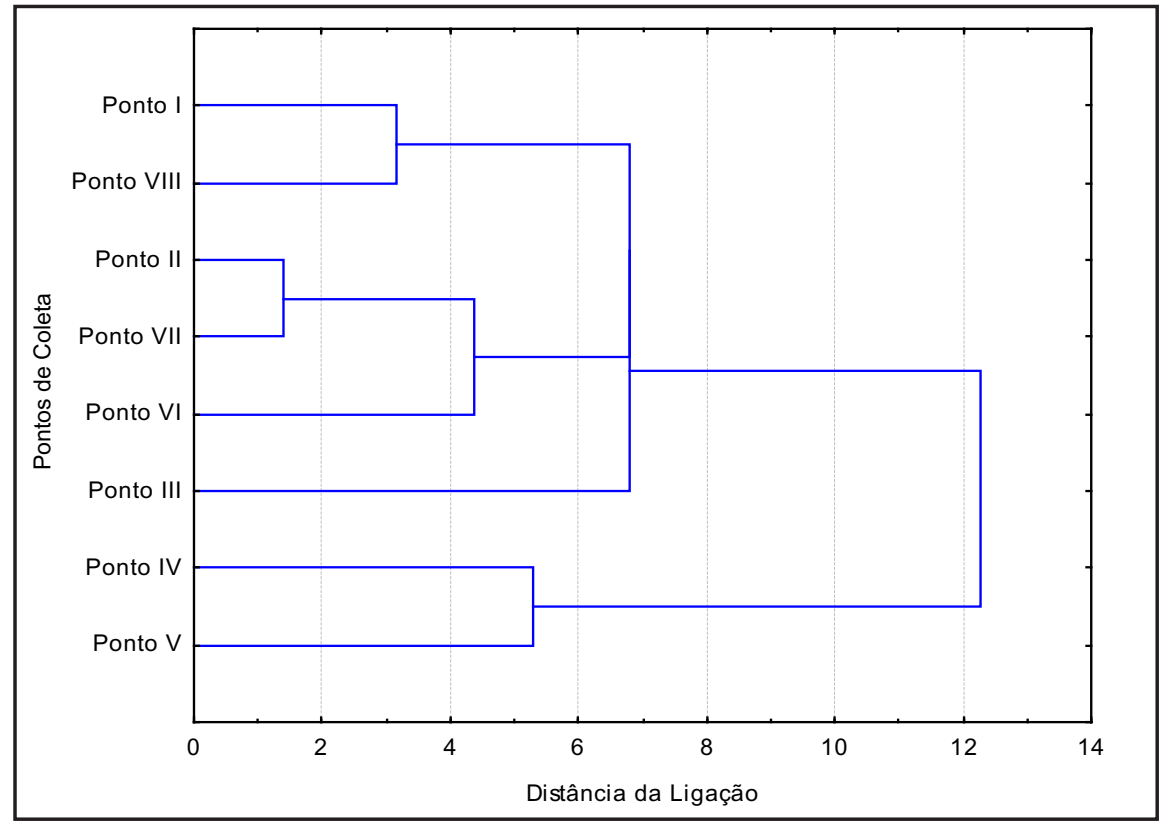

Figura 6. Diagrama de árvore da família de Staphylinidae entre os oito pontos de coleta, baseado na análise Cluster (Complete Linkage), no período de abril de 2008 e março de 2009, no IZMA, Eng. Paulo de Frontin - RJ.

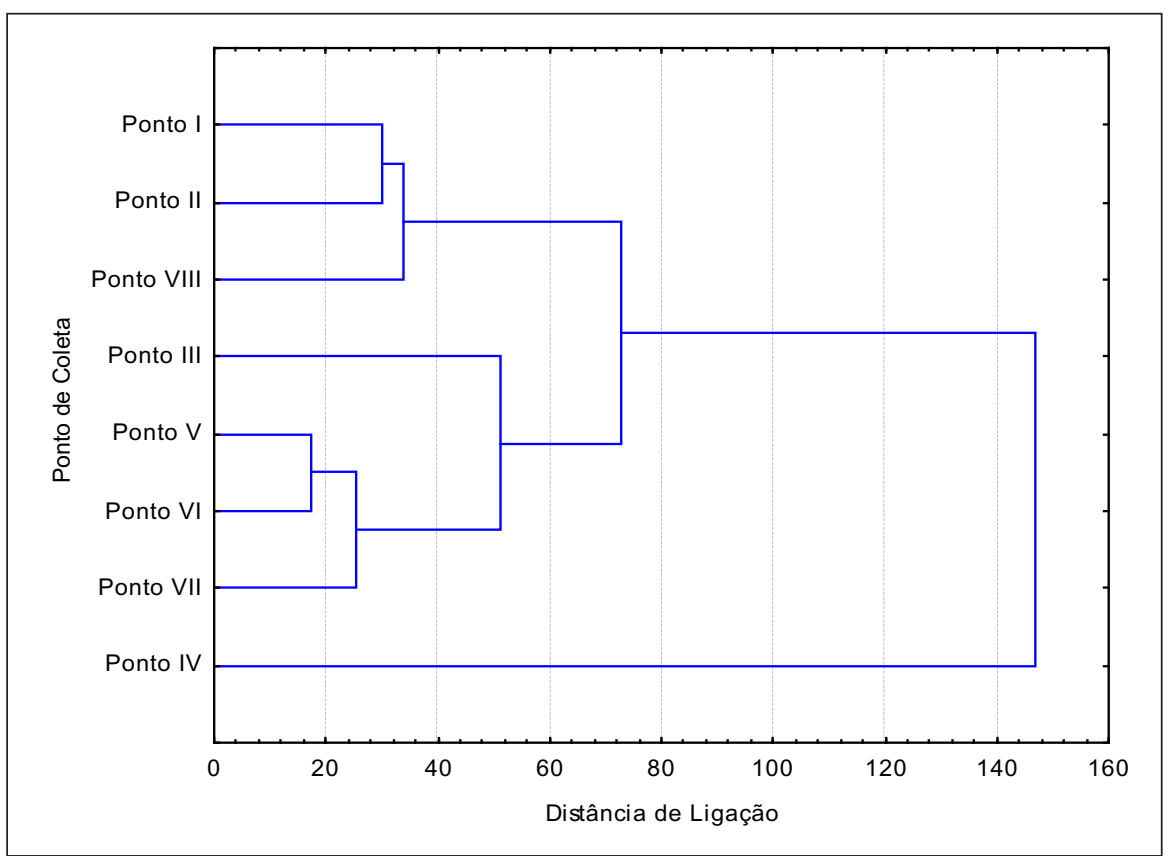

Figura 7. Diagrama de árvore das demais famílias de Coleoptera entre os oito pontos de coleta, baseado na análise Cluster (Complete Linkage), no período de abril de 2008 e março de 2009, no IZMA, Eng. Paulo de Frontin - RJ. 
Os pontos I e III apresentaram maior Diversidade (0,54), enquanto o menor valor foi encontrado no ponto V $(0,43)$; pois há correlação direta com os índices de Equitabilidade, também apresentando os maiores valores nos pontos I e III $(0,65)$, tendo o menor no ponto IV $(0,49)$ (Figura 8$)$. Já o índice de Dominância é inversamente proporcional à Diversidade, com os pontos I e III apresentando os menores valores $(0,46)$, e o ponto V apresentando o maior valor $(0,57)$ (Figura 9). Quanto à Similaridade, observou-se maior ligação entre os pontos III e VII (Figura 10), os mais abundantes depois do ponto IV, ficando distante destes por apresentar uma frequência muito maior em relação a todos os pontos, esse fato também explica o menor valor de equitabilidade neste ponto, e consequentemente baixo índice de diversidade.

Diversos autores em seus estudos, citam diferenças entre áreas de borda, ambientes com diferentes níveis de fragmentação ou cobertura vegetal (Pohl et al. 2007; Gardner et al. 2008; Cagnolo et al. 2009; Sobek et al. 2009), nos quais a maior cobertura vegetal ou complexidade sustentam comunidades mais estáveis e/ou diversas (Eisenhauer et al. 2008). Assim, os dados encontrados no presente estudo corroboram com estes autores, pois nos locais mais estáveis no fragmento estudados verifica-se maior incidências de Silphidae e Staphylinidae.

Em relação às coletas, a $1^{\mathrm{a}}$ coleta apresentou maior dominância $(0,53)$, sendo Staphylinidae a família dominante, e menor diversidade $(0,47)$; enquanto a $4^{\text {a }}$ coleta apresentou maior diversidade $(0,55)$ e menor dominância $(0,45)$ (Figura 11$)$, pois as famílias mais abundantes, Staphylinidae e Silphidae, apresentaram uma abundância aproximada nesta coleta. Segundo Rocha et al. (2010), o uso de bioindicadores é essencial para o monitoramento ambiental. As principais características de um bioindicador são: riqueza e diversidade de espécies, de fácil manuseio, fidelidade ecológica, a fragilidade a pequenas mudanças ambientais e as respostas organismo bom. A Classe Insecta tem todos eles. No entanto, algumas espécies respondem melhor que outras a essas mudanças e de acordo com o meio ambiente.

Não se pode descartar que a metodologia utilizada para coleta limitou a biodiversidade capturada, pois foi usado apenas um tipo de armadilha. Chung et al., (2000) comentaram haver uma diferença na composição de famílias dependendo da forma de coleta, com cada método amostrando uma fauna específica. O fato de a última coleta ter sido no final do verão/início do outono, em vez de realizada no mês central do verão, também influenciou os índices de diversidade e abundância total, pois algumas famílias tem maior atividade nessa estação do ano.

Ainda em seus estudos Rocha et al., (2010) concluíram que a classe Insecta tem muitos representantes potenciais que podem ser usados como bioindicadores ambientais, entre os quais algumas espécies das ordens Coleoptera, Diptera, Lepidoptera, Hymenoptera, Hemiptera, Isoptera e outros. E apesar do presente estudo utilizar-se de famílias para estimar a qualidade ambiental, as prováveis morfoespécies capturadas pertencem a mesma família o que podem ser utilizadas como indicadores de qualidade. Da mesma forma Beiroz et al., (2010) afirma que as famílias Staphylinidae, Nitidulidae, Scarabaeidae e Scolytidae podem ser considerados como grupos bioindicadores, segundo este estudo, pois estiveram presentes em todos os pontos de coleta, ao longo de todo ano, além de serem as mais abundantes. 
Staphylinidae e Silphidae (Coleoptera) como Potenciais Famílias Bioindicadoras de Qualidade Ambiental

Fabiana dos Santos Fernandes - Sueyla da Silva Alves - Hélio Freitas Santos - William Costa Rodrigues

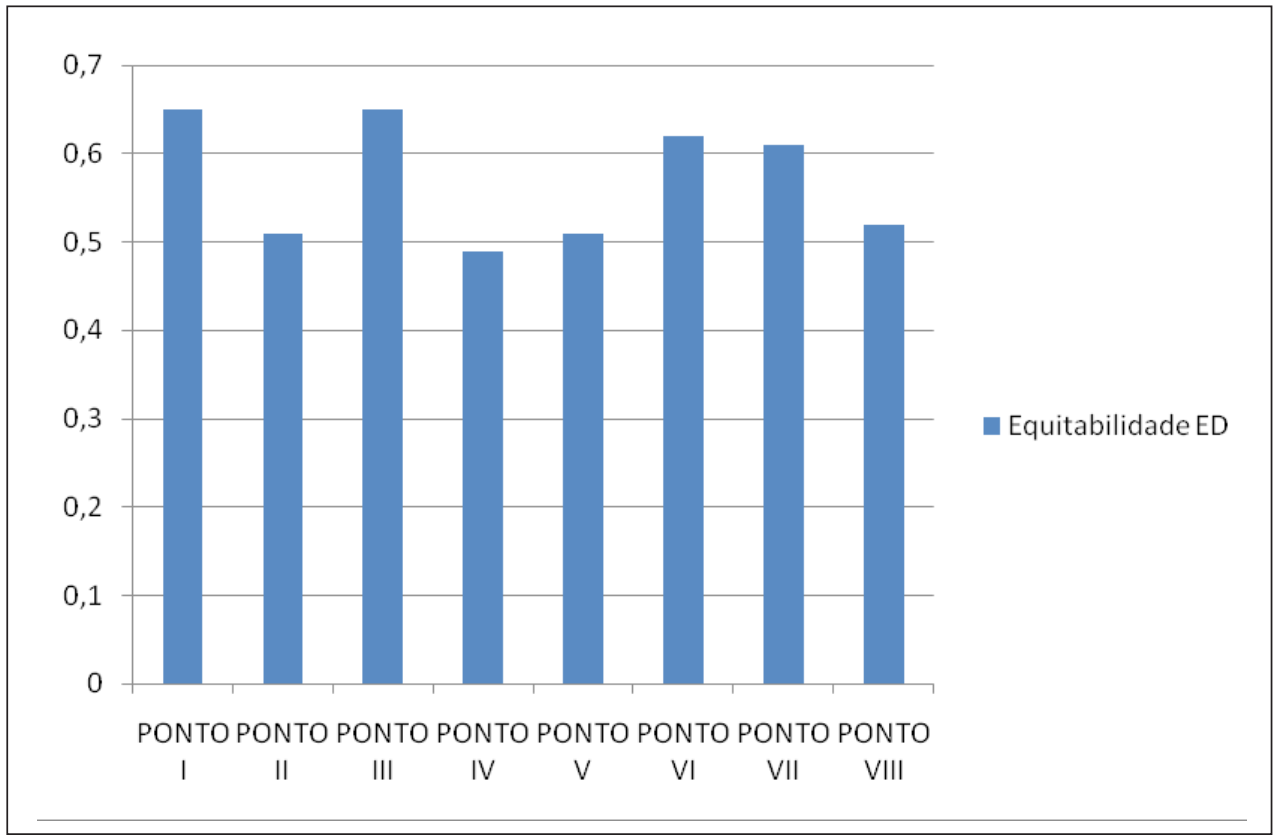

Figura 8. Equitabilidade ED (Simpson) das famílias de Coleoptera por ponto, no período de abril de 2008 e março de 2009, no IZMA, Eng. Paulo de Frontin - RJ.

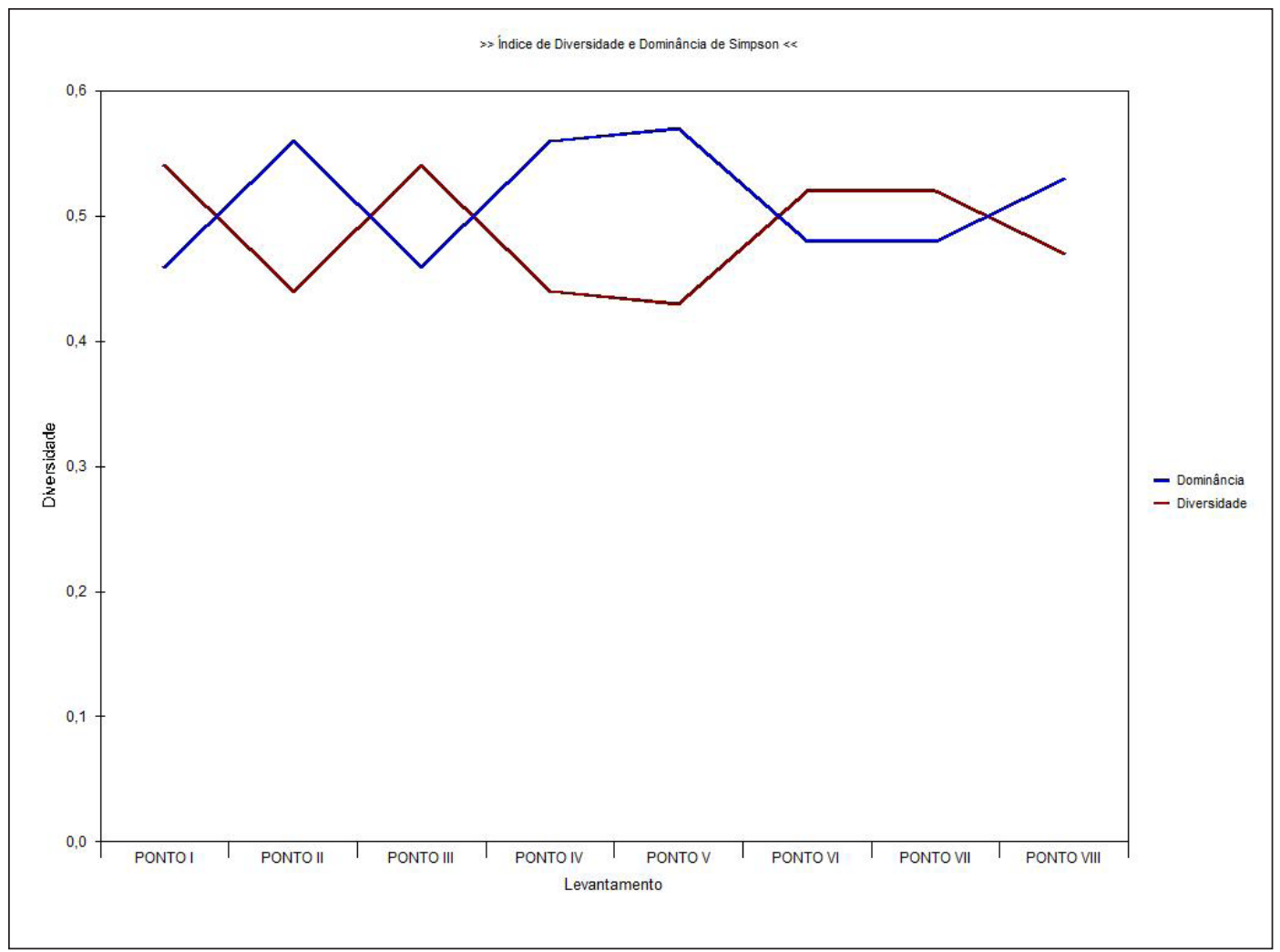

Figura 9. Diversidade e Dominância de Simpson das famílias de Coleoptera por ponto, no período de abril de 2008 e março de 2009, no IZMA Eng. Paulo de Frontin - RJ. 
Staphylinidae e Silphidae (Coleoptera) como Potenciais Famílias Bioindicadoras de Qualidade Ambiental

Fabiana dos Santos Fernandes - Sueyla da Silva Alves - Hélio Freitas Santos - William Costa Rodrigues

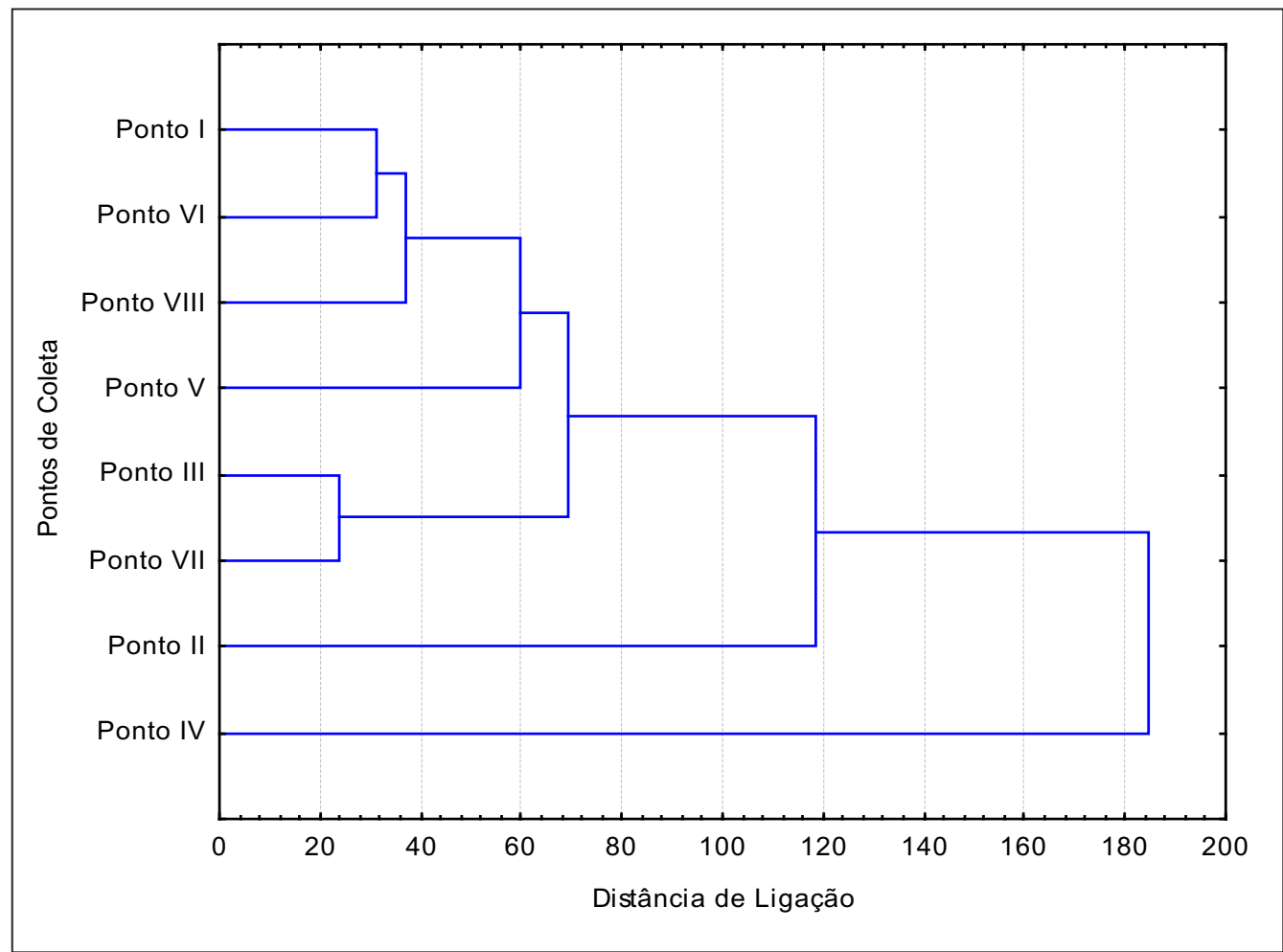

Figura 10. Diagrama de árvore entre pontos de coleta, baseado na análise Cluster (Complete Linkage), no período de abril de 2008 e março de 2009, no IZMA, Eng. Paulo de Frontin - RJ.

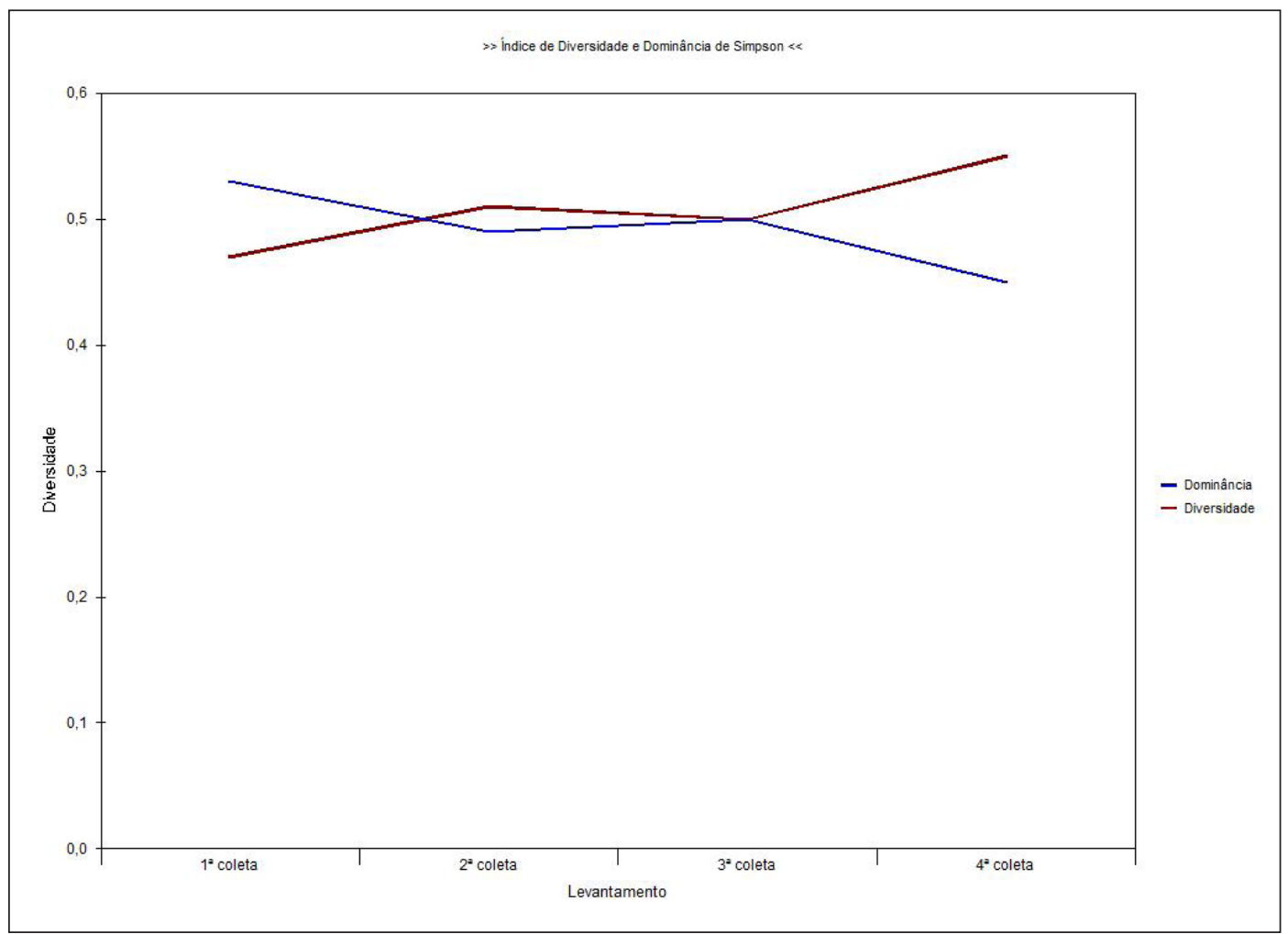

Figura 11. Diversidade e Dominância de Simpson das famílias de Coleoptera por coleta, no período de abril de 2008 e março de 2009, no IZMA, Eng. Paulo de Frontin - RJ. 


\section{Conclusão}

Apesar de haver muitos pesquisadores e publicações na área de bioindicadores no Brasil, as pesquisas ainda se encontram em estágio inicial. Desta forma, muitas questões básicas estão abertas e poucas generalizações podem ser feitas.

O presente trabalho confirma que estudos em nível de família podem ser usados em levantamentos com objetivos de avaliar a qualidade ambiental.

Pôde ser observada a sensibilidade das famílias Staphylinidae, e Silphidae em relação à composição florística, nível de serrapilheira, e luminosidade. Enquanto Staphylinidae parece preferir áreas de interior de mata, com maior quantidade de serrapilheira (NS3); Silphidae apresentou uma tendência a áreas intermediárias, com nível médio de serrapilheira (NS2), e temperaturas mais elevadas o que aponta essas famílias como potenciais bioindicadoras de qualidade ambiental.

Seus hábitos alimentares, sendo a maioria detritívoros, sugerem que os pontos III, IV, VI, e VII são os mais conservados, por apresentarem maior abundância dessas famílias nessas áreas. Sendo necessário identificá-los posteriormente em espécie, para confirmar seus grupos tróficos, chegando então a espécies bioindicadoras.

A sazonalidade também se mostrou como importante fator, interferindo na abundância, e consequentemente na dominância das famílias de coleópteros.

Sabendo-se que o município de Eng. Paulo de Frontin tem sua área coberta por aproximadamente 52\% de Floresta Atlântica, e que pouco se sabe sobre sua fauna, principalmente a entomofauna bioindicadora deste bioma, este estudo mostrou sua importância por intermédio dos resultados encontrados. Mostrando também ser importante continuar realizando estudos com objetivo de conhecer e entender a dinâmica do bioma local. 


\section{Referências}

Beiroz, W., Zaú, A.S., Castro Jr., E. (2010). Impacto das Estradas na Distribuição de Besouros em um Fragmento de Mata Atlântica de Encosta no Parque Nacional da Tijuca, Rio de Janeiro, RJ. EntomoBrasilis, 3, 64-68. Disponível em: <www.periodico. ebras.bio.br/ojs >

Brower, J.E.; Zar, J.H. \& von Ende, C.N. (1997) Field and Laboratory Methods for General Ecology. (4 ${ }^{\text {th }}$ ed.). New York: WCB Mc-Graw-Hill

Brown, K.S.Jr. (1991). Conservation of Neotropical Environments: Insects as Indicators. In: Collins, N.M., Thomas, J.A. (Eds.). The conservation of insects and their habitats. London: Academic Press.

Buzzi, Z.J., Miyazaki, R.D. (1999). Entomologia Didática. Curitiba: Universidade Federal do Paraná.

Cagnolo, L., Valladares, G., Salvo, A., Cabido, M., Zak, M. (2009). Habitat Fragmentation and Species Loss across Three Interacting Trophic Levels: Effects of Life History and Food-Web Traits. Conservation Biology. 23, 1167-1175.

Chung, A.Y., Eggleton, P., Speight, M.R., Hammond, P.M., Chey, V.K. (2000). The diversity of beetle assemblages in different habitat types in Sabah, Malaysia. Bulletin of Entomological Research, 90, 475-496.

Correia, M.E.F., Pinheiro, L.B.A. (1999). Monitoramento da fauna de solo sob diferentes coberturas vegetais em um sistema integrado de produção Agrícola, Seropédica, R.J. Seropédica: EMBRAPA Agrobiologia.

Costa Lima, A. (1952). Insetos do Brasil - Série Didática. Rio de Janeiro: Escola Nacional de Agronomia.

Dale, V.H., Beyeler, S.C. (2001). Challenges in the development an use of ecological indicators. Ecological Indicators, 1, 1, 3-10.

Dummel, K., Oliveira, E.A., Zardo, C.M.L. D’Incao, F. (2011). Variação de Abundância, Diversidade Ecológica e Similaridade de Coleoptera (Insecta) entre Restinga e Marisma do Estuário da Lagoa dos Patos, Rio Grande, RS. EntomoBrasilis, 4, 39-44. Disponível em: <www.periodico.ebras.bio.br/ojs >

Dunxião, H., Chunru, H., Yaling, X., Banwang, H., Liyuan, H., Paoletti, M.G. (1999). Relationship between soil arthropods and soil properties in a Suburb of Qianjiang City, Hubei, China. Critical Rewiews in Plant Sciences, 18, 3, 467-473.

Eisenhauer, N., Milcu, A., Sabais, C.W., Scheu, S. (2008). Animal Ecosystem Engineers Modulate the Diversity-Invasibility Relationship. Public Library of Science ONE, 3, e3489.

Freitas, A.V.L., Leal, I.R., Uehara-Prado, M., Iannuzzi, L. (2005). Insetos como indicadores de conservação da paisagem. In: Rocha, C.F.D., Bergallo, H.G., Van Sluys, M., Alves, M.A.S. (orgs.). Biologia da conservação. Rio de Janeiro: UERJ.

Gardner, T. A., Hernández, M.I.M., Barlow, J., Pere, C.A (2008). Understanding the biodiversity consequences of habitat change: the value of secondary and plantation forests for neotropical dung beetles. Journal of Applied Ecology. 45, 883-893. 
Iannuzzi, L., Maia, A.C.D., Nobre, C.E.B., Suzuki, D.K., Muniz, F.J.A. (2003). Padrões locais de diversidade de Coleoptera (Insecta) em vegetação de Caatinga, p. 367-389. In: Leal, I.R., M. Tabarelli \& J.M.C. Silva. (Org.). Ecologia e conservação da caatinga. Editora Universitária da Universidade Federal do Pernambuco, Recife, 822p.

Knoepp, J.D., Coleman, D.C., Crossey Jr D.A., Clark, J.S. (2000). Biological indices of Soil quality: an ecosystem case study of their use. Forest Ecology and Management, 138, 357- 368 .

Lopes, B.G.C. (2008). Levantamento da entomofauna bioindicadora da qualidade ambiental em diferentes áreas do Alto Jequitinhonha - Minas Gerais. 2008. 47f. Trabalho de Conclusão de Curso - Escola Agrotécnica Federal de Inconfidentes. Inconfidentes, Minas Gerais.

Marinoni, R.C., Dutra, R.R.C. (1997). Famílias de Coleoptera capturados com armadilhas Malese em oito localidades do estado do Paraná, Brasil. Diversidades alfa e beta. Revista Brasileira de Zoologia, 14, 3, 751-770.

Marinoni, R.C., Ganho, N.G. (2003). Fauna de Coleoptera no Parque Estadual de Vila Velha, Ponta Grossa, Paraná, Brasil. Revista Brasileira de Zoologia, 20, 4, 737-744.

Milhomem, M.S., Vaz-de-Mello, F.Z., Diniz, I.R. (2003). Técnicas de coleta de besouros copronecrófagos no cerrado. Brasília: Pesquisa Agropecuária Brasileira.

Nicholsa, E., Larsenb, T., Spectora, S., Davise, A.L., Escobarc, F., Favilad, M., Vulinece, K. (2007). Global dung beetle response to tropical forest modification and fragmentation: A quantitative literature review and meta-analysis. Biological Conservation, 137, 119.

Odum, E.P. (1983). Ecologia. Rio de Janeiro: Editora Guanabara.

Petroni, D.M. (2008). Diversidade de famílias de Coleoptera em diferentes fragmentos florestais no município de Londrina, Pr - Brasil. Dissertação (Mestrado em Ciências Biológicas) - Universidade Estadual de Londrina.

Pohl, G.R., Langor, D.W., Spence, J.R. (2007). Rove beetles and ground beetles (Coleoptera: Staphylinidae, Carabidae) as indicators of harvest and regeneration practices in western Canadian foothills forests. Biological Conservation, 137, 294-307.

Rocha, J.R.M., Almeida, J.R., Lins, G.A., Durval, A. (2010). Insects as Indicators of Environmental Changing and Pollution: A Review of Appropriate Species and their Monitoring. HOLOS Environment, 10, 250-262.

Rodrigues, W.C. (2005). DivEs - Diversidade de espécies. Versão 2.0. Software e Guia do Usuário. Disponível em: <http://www.ebras.bio.br/dives>.

Santos, B.B., Silva, S.S. (2004). Coleopterofauna de uma área semicaducifólia e de pastagem de Goiânia, Goiás, Brasil. Brasília: XXV Congresso Brasileiro de Zoologia: Resumos.

Sobek, S., Steffan-Dewenter, I., Scherber, C., Tscharntke, T. (2009). Spatiotemporal changes of beetle communities across a tree diversity gradient. Diversity and Distributions, 15, 660-670.

Stork, N.E., Eggleton, P. (1992). Invertebrates as determinants and indicators of soil quality. American Journal of Alternative Agriculture, 7, 38-47, 199. 
Tylianakis, J., Veddeler, D., Lozada, T., López, R.M., Benítez, P., Klein, A.M.; Koning, G.H.J., Olschewski, R., Veldkamp, E., Navarrete, H., Onore, G., Tscharntke, T. (2007). Biodiversity of land-use systems in coastal Ecuador and bioindication using trapnesting bees, wasps, and their natural enemies. Lyonia. 6, 7-15.

Urbini, A.; Sparvoli, E.; Turillazzi, S. (2006) Social paper wasps as bioindicators: a preliminary research with Polistes dominulus (Hymenoptera: Vespidae) as a trace metal accumulator. Chemosphere, 64, 697-703.

Wink, C., Guedes, J. V. C., Fagundes, C. K., Rovedder, A. P.(2005). Insetos como indicadores da qualidade ambiental. Revista de Ciências Agroveterinárias, 4, 1, 6070 . 